\title{
IS THE ANOMALOUS MICROWAVE EMISSION DUE TO THE ROTATION OF INTERSTELLAR PAHS? PLANCK ${ }^{1}$ RESULTS; PLANCK - AKARI PROJECT
}

\author{
Planck Collaboration, M. Giard ${ }^{1,2}$, O. Berné ${ }^{1,2}$, Y. Doi ${ }^{3}$, D. Ishihara ${ }^{3}$, Ch. Joblin ${ }^{1,2}$, I. Kaneda $^{4}$, \\ D. Marshall ${ }^{1,2}$, T. Nakagawa ${ }^{6}$, R. Ohsawa ${ }^{3}$, T. OnakA ${ }^{3}$, I. Sakon ${ }^{3}$, H. Shibai ${ }^{3}$, And N. Ysard ${ }^{5}$ \\ ${ }^{1}$ Université de Toulouse; UPS-OMP; IRAP; Toulouse, France \\ ${ }^{2}$ CNRS; IRAP; 9 Av. colonel Roche, BP 44346, F-31028 Toulouse cedex 4, France \\ ${ }^{3}$ University of Tokyo, Bunkyo-ku, 113-0033, Tokyo, Japan \\ ${ }^{4}$ Nagoya University, Chikusa-ku, 464-8602, Nagoya, Japan \\ ${ }^{5}$ Institut dAstrophysique Spatiale, CNRS (UMR8617) Université Paris-Sud 11, Batiment 121, Orsay, France \\ ${ }^{6}$ Sagamihara, 252-5210, Kanagawa, Japan \\ E-mail: martin.giard@irap.omp.eu \\ (Received July 13, 2012; Accepted August 16, 2012)
}

\begin{abstract}
We show how the rotation emission from isolated interstellar Polycyclic Aromatic Hydrocarbons (PAHs) can explain the so-called anomalous microwave emission (AME). AME has been discovered in the last decade as microwave interstellar emission (10 to $70 \mathrm{GHz}$ ) that is in excess compared to the classical emission processes: thermal dust, free-free and synchrotron. The PAHs are the interstellar planar nano-carbons responsible for the near infrared emission bands in the 3 to 15 micron range. Theoretical studies show that under the physical conditions of the interstellar medium (radiation and density) the PAHs adopt supra-thermal rotation velocities, and consequently they are responsible for emission in the microwave range. The first results from the PLANCK ${ }^{1}$ mission unexpectedly showed that the AME is not only emitted by specific galactic interstellar clouds, but it is present throughout the galactic plane, and is particularly strong in the cold molecular gas. The comparison of theory and observations shows that the measured emission is fully consistent with rotation emission from interstellar PAHs. We draw the main lines of our PLANCK-AKARI collaborative program which intends to progress on this question by direct comparison of the near infrared (AKARI) and microwave (PLANCK) emissions of the galactic plane.
\end{abstract}

Key words: interstellar molecules; infrared and microwave emission

\section{INTRODUCTION}

The Differential Microwave Radiometer (DMR) aboard the NASA COBE satellite provided the first full sky and high sensitivity microwave maps of the galactic emission at $31.5,53$ and $90 \mathrm{GHz}$. In a detailed cross-

\footnotetext{
1 Based on observations obtained with Planck (http://www.esa.int/Planck), an ESA science mission with instruments and contributions directly funded by ESA Member States, the USA, and Canada.
}

correlation analysis of the $4 \mathrm{yr}$ maps with the $408 \mathrm{MHz}$ synchrotron emission and the far infrared $140 \mu \mathrm{m}$ dustdominated emission from the Diffuse Infrared Background Experiment (DIRBE) aboard COBE, Kogut et al. (1996) showed that the dust correlated component detected at DMR frequencies implies a dust spectral in$\operatorname{dex} \beta=1.1$ running from $140 \mu \mathrm{m}$ to $6 \mathrm{~mm}$. Although the authors did not interpret their result in a physical manner, this was probably one of the first detections 


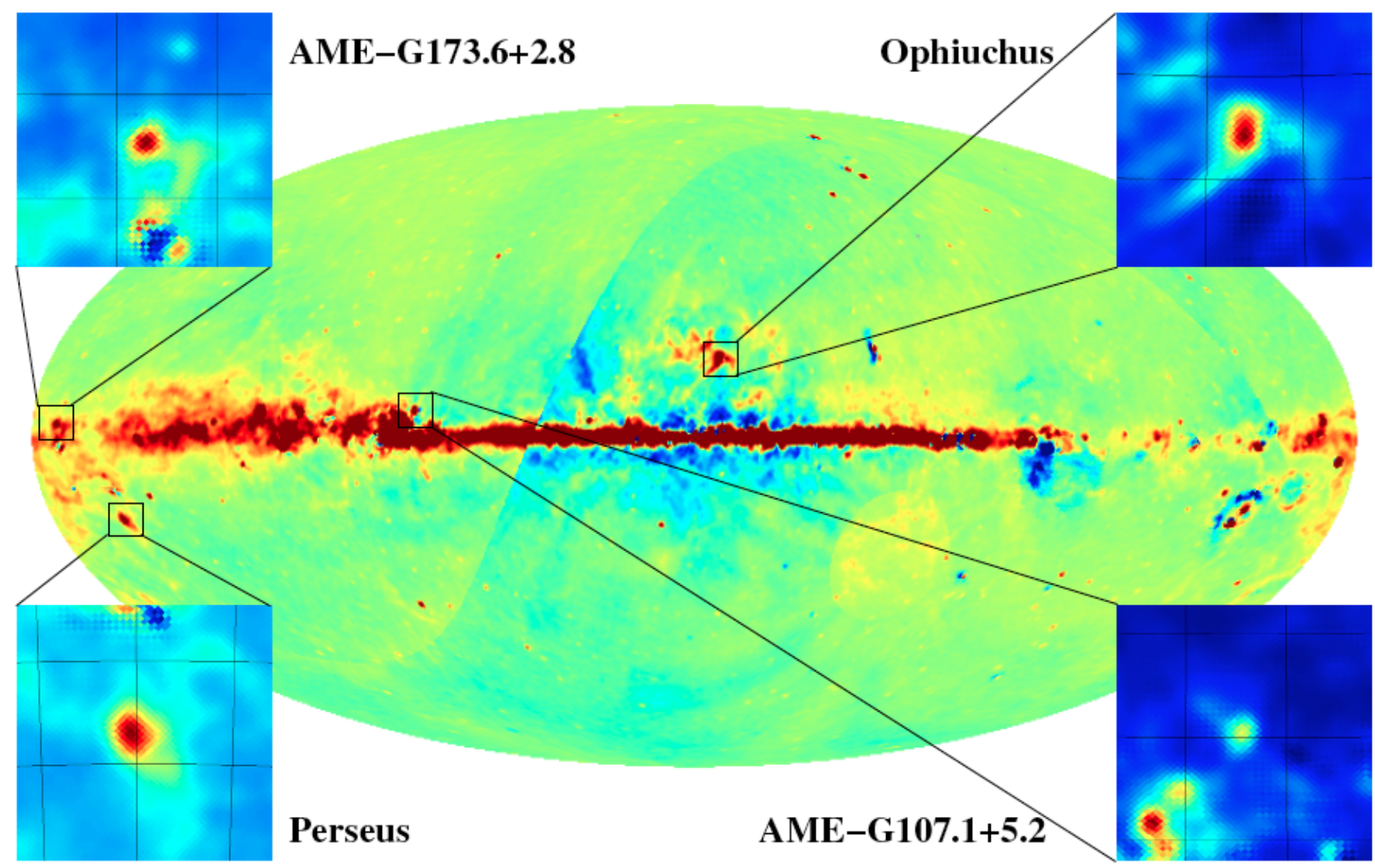

Fig. 1. Residuals in the full sky Planck LFI $28.5 \mathrm{GHz}$ one degree smoothed map after subtraction of synchrotron, free-free and thermal dust emission. $12.5 \times 12.5$ degree cut out maps are shown for four peculiar regions including the Perseus and $\rho$ Ophiuchi molecular clouds; reproduced from Planck Collaboration 2011a "Planck early results XX : New light on anomalous microwave emission from spinning dust grains."

of a microwave galactic emission correlated to dust and which is above what can be expected from thermal dust emission with $\beta=2$. A similar result was obtained in the statistical analysis of ground based data from the CMB Saskatoon experiment in the same frequency range by De Oliveira-Costa et al. (1997). Leitch et al. (1997) clearly identified such an excess in a peculiar cirrus cloud toward the north celestial pole using the Owens Valley telescope at 14.5 and $32 \mathrm{GHz}$, and they were the first to name it "the anomalous component of galactic emission".

Since then, this excess has been referred to as the Anomalous Microwave Emission (AME) because its physical origin was not trivial. It could simply have been explained by free-free emission from dust correlated ionized gas. However, at that time the observation of the $H \alpha$ emission at high galactic latitude was very limited in coverage and sensitivity. The existence and the origin of the AME was extensively de- bated at the IAS-Princeton CMB meeting organized in November 1998 by A. de Oliveira-Costa and M. Tegmark (1999). In the mean time, Draine and Lazarian (1998) had published a detailed model of microwave emission from rapidly rotating interstellar dust grains which could explain the AME. Athough they had no definite proof the AME could not be free-free emission, Kogut (1999) and De Oliveira-Costa et al. (1999) examined different ground based data sets and were the first to assign the AME to the emission of spinning dust grains.

With the availability of the full sky high sensitivity $H \alpha$ surveys (SHASSA for the southern sky, see Gaustad et al., 2001; WHAM for the northern sky, see Reynolds et al., 1998) it then became clear that the AME was not correlated with the free-free emission. Finkbeiner et al. (2002) performed a dedicated study of several high latitude dust clouds using the 43 meter NRAO telescope at 5,8 and $10 \mathrm{GHz}$ and they were 
able to demonstrate that the microwave spectrum of the AME is fully consistent with emission from spinning dust grains as predicted by Draine and Lazarian (1998). Banday et al. (2003) and Lagache (2003) concluded the same on the basis of full sky studies of DMRCOBE and WMAP data respectively. Lagache (2003) was among the first to propose that the AME could be rotating emission from transiently heated very small particles of nanometric size, either of 3D (very small grains) or 2D (polycyclic aromatic hydrocarbons, eg $\mathrm{PAH})$ geometries.

The theory of low frequency radio emission from spinning dust grains was originally proposed in different astrophysical contexts by Erickson (1957) and Martin (1972). Rouan et al. (1992) established that similarly to dust grains which are raised to supra-thermal rotation velocities by the radiative and collisional processes at work in the insterstellar medium, PAHs are actually raised to supra-thermal rotation velocities by the various excitation processes (radiative and collisional), implying the emission of rotation photons in the gigaHertz range for a typical PAH made of 78 carbon atoms. Since the original work of Draine and Lazarian (1998), two other detailed models of the microwave emission from very small grains and/or PAH molecules have been developed which can be used to compare to the data in the AME frequency range (1 to $30 \mathrm{GHz}$ ). The latest developments can be found in the three following references: Ysard and Verstraete (2010), Hoang, Drain and Lazarian (2010), and Silsbee et al. (2011).

\section{PLANCK RESULTS}

With nine frequency bands covering the electromagnetic spectrum from 28.5 to $857 \mathrm{GHz}$, PLANCK (see Tauber et al. 2011 "Planck pre-launch status : The Planck mission") is the ideal tool to measure all the microwave interstellar emissions in a single shot, once the CMB sky has been subtracted : thermal dust dominating at high frequencies, free-free, AME and synchrotron at low frequencies.

In Planck Collaboration (2011a) we have obtained a full sky map of the residuals at $28.5 \mathrm{GHz}$ after subtraction of the CMB and simple templates for the synchrotron (extrapolated from the $408 \mathrm{MHz}$ survey from Haslam et al. 1982), free-free (SHASSA and WHAM $H \alpha$ surveys corrected for dust absorption as in Dickinson et al., 2003) and thermal dust (extrapolated from the Planck maps at 545 and $143 \mathrm{GHz}$ ). This map is re-
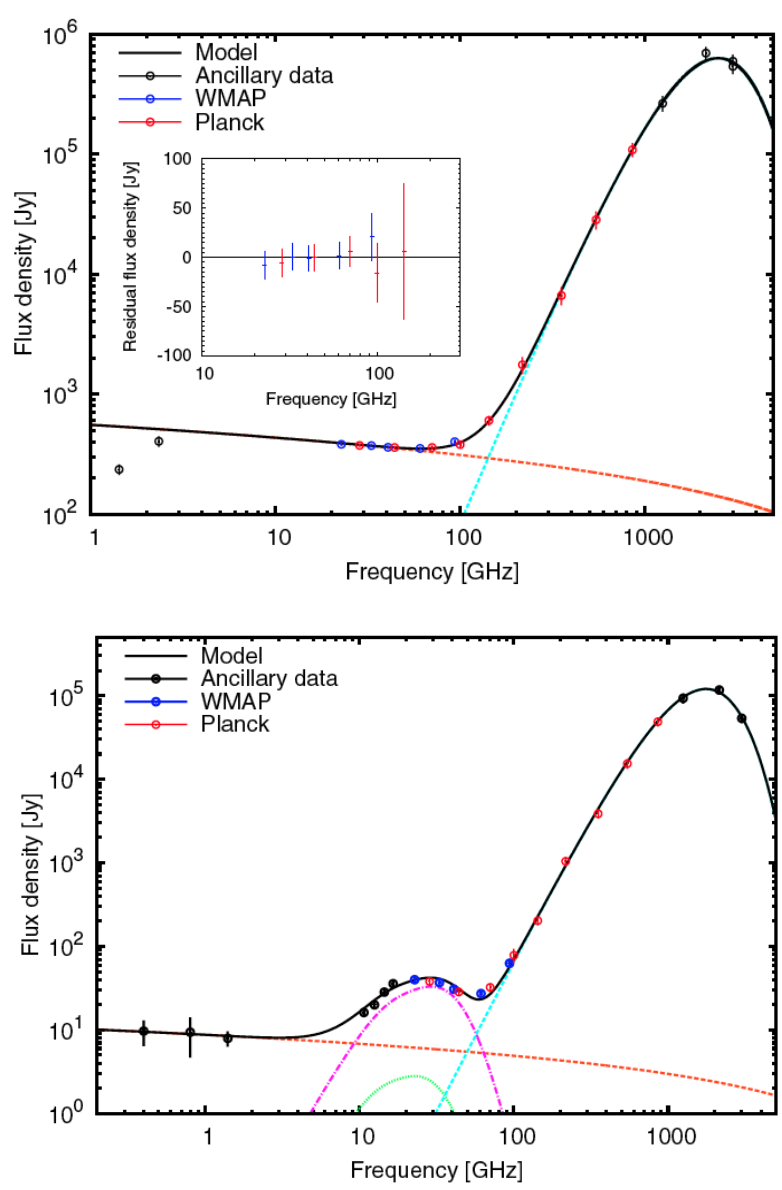

Fig. 2. Spectral energy distributions (SED) of a pure HII region in M42 (upper) and the AME region in the Perseus molecular cloud (lower); The model is a sum of free-free (orange dashed line), thermal dust (light blue dashed line) and spinning PAHs embedded in dense molecular gas for the AME bump in the 10 to $40 \mathrm{GHz}$ range (magenta dot-dashed line); reproduced from Planck Collaboration 2011a "Planck early results XX : New light on anomalous microwave emission from spinning dust grains."

produced in Figure 1. Although there are some obvious artifacts due to the assembly of different surveys used in the production of the subtracted templates, and despite the fact that the inner galactic plane region is certainly not reliable because of the very high optical thickness of the $H \alpha$ emission, this map shows several potential AME regions, some already known (eg in Perseus and $\rho$ Ophiuchi molecular clouds), and some new ones (AME labels).

Figure 2 shows the spectral energy distributions (SED) derived respectively for a pure HII region (M42, 

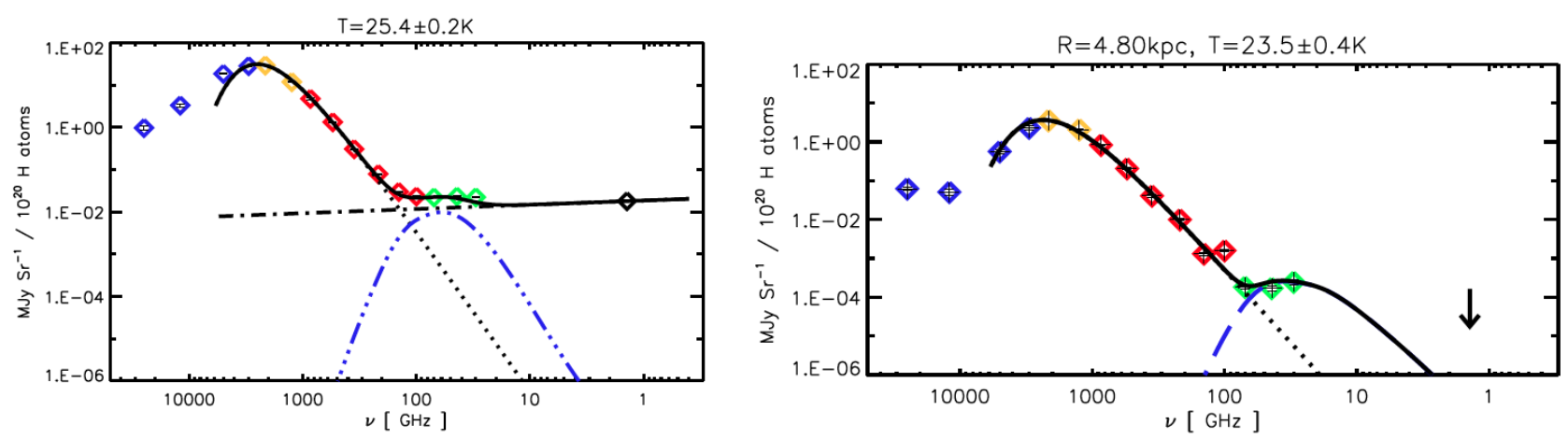

Fig. 3. SEDs of the HII gas in the galactic disk (left) and the molecular gas in the inner galaxy (right); the data are from IRAS (blue), DIRBE (yellow), PLANCK-HFI (red) and PLANCK-LFI (green); the model includes thermal dust (temperature as labeled), free-free for the HII component, and spinning PAHs; reproduced from Planck Collaboration 2011b "Planck early results. XXI : Properties of the interstellar medium in the Galactic plane"

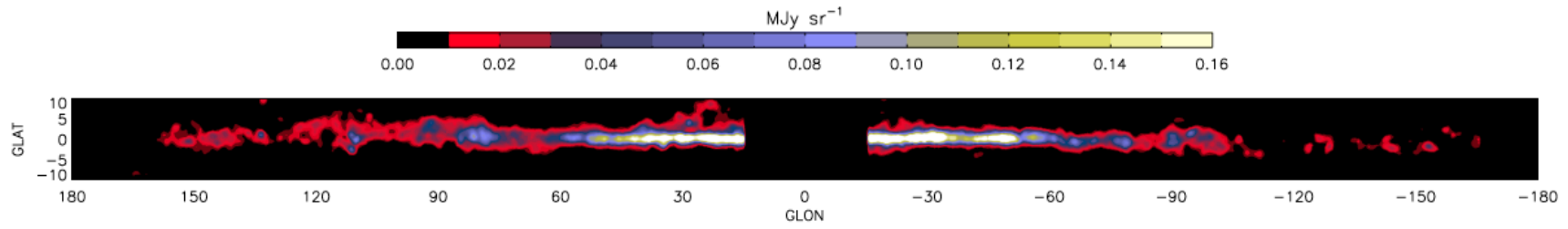

Fig. 4. Spinning dust emission at $28.5 \mathrm{GHz}$ in the Galactic plane in MJy/sr; reproduced from Planck Collaboration 2011b "Planck early results. XXI : Properties of the interstellar medium in the Galactic plane"

upper) and for the AME region in the Perseus molecular cloud (lower) where Davies et al. (1987) performed what is likely to be the first historical detection of the AME. For the HII region, the SED is fully consistent with pure free-free plus thermal dust, and for the AME region in Perseus there is a clear bump in the SED in the 10 to $40 \mathrm{GHz}$ range in addition to the free-free and thermal dust contributions. The AME SED bump is for the first time very well delineated with the combination of ground based and space based data. The AME SED is very well fitted by emission from spinning six Angstróm size PAHs molecules embedded in the dense molecular gas of this region (magenta dotdashed line). The model used to compute the emission from spinning PAHs is SPDUST (case 2) from Silsbee et al. (2011). This study clearly demonstrates that the spinning PAH emission cannot arise from low density atomic gas (green dotted line in Figure 2 lower frame).

In Planck Collaboration (2011b) we have derived the SEDs of the different phases of the interstellar medium (HII, HI and H2) within the galactic plane combining the IRAS, DIRBE and PLANCK data sets, and correlating each of them with templates tracing the dif- ferent gas phases (WMAP free-free for HII, $21 \mathrm{~cm}$ for $\mathrm{HI}$, and CO line for H2). Figure 3 shows two sample SEDs obtained in this decomposition: for the HII component (left), and for the molecular component in the inner galactic disk, the so-called "molecular ring" (right). The rotation emission of PAHs is marginally visible in the HII component, and it dominates the low frequency end of the SED in the case of the molecular component. In both cases we have used a standard PAH abundance relative to the gas. The main result of this study is that the model of rotation emission from free spinning $\mathrm{PAH}$ molecules has been able to predict the PLANCK-LFI fluxes in all phases of the galactic interstellar matter using the standard PAH abundance and size range.

Finally, we show in Figure 4 our estimation of the AME (spinning dust emission) contribution to the 28.5 $\mathrm{GHz}$ map of the galactic plane.

\section{AKARI - PLANCK PROSPECTS}

The availability of high quality AKARI maps of the full sky, as it has been shown in this 2nd AKARI meeting, 


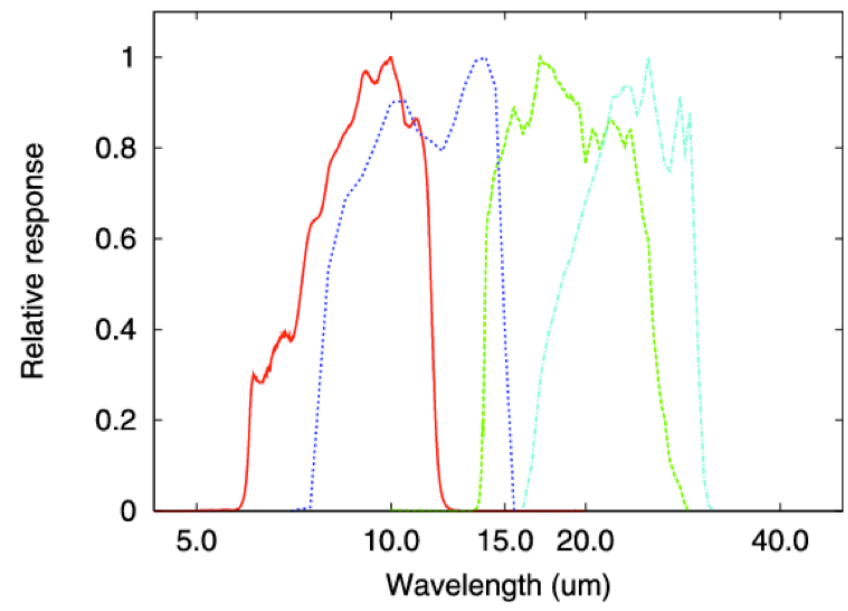

Fig. 5. AKARI MIR-S and MIR-L filter bands compared to the IRAS 12 and $25 \mu \mathrm{m}$ bands.

opens new possibilities to test the PAH hypothesis as being at the origin of the AME. The two AKARI MIR wide band filters (MIR-S and MIR-L), together with the IRAS 12 and $25 \mu \mathrm{m}$ filters, make a set of four nondegenerate wavelength bands which should allow to retrieve the intensity of the wider PAH infrared vibration bands ( 7.7 and $11.3 \mu \mathrm{m}$ ) and the underlying continuum (see Figure 5). Our plan is to complement our SEDs with this data sets so that we can test the hypothesis that the PAHs are responsible of the AME (rotation) and of the infrared bands (vibration) on isolated clouds and for the different phases of the interstellar medium. The PAHs abundance has been shown to vary from place to place, depending on the intensity and hardness of the radiation field. If the PAH hypothesis is true, we should then be able to correlate the variation of the AME with the intensity of the infrared bands.

\section{ACKNOWLEDGEMENTS}

Planck is a project of the European Space Agency - ESA - with instruments provided by two scientific Consortia funded by ESA member states (in particular the lead countries: France and Italy) with contributions from NASA (USA), and telescope reflectors provided in a collaboration between ESA and a scientific Consortium led and funded by Denmark. The development of Planck has been supported by: ESA; CNES and CNRS/INSU-IN2P3-INP (France); ASI, CNR, and INAF (Italy); NASA and DoE (USA); STFC and UKSA (UK); CSIC, MICINN and JA (Spain); Tekes, AoF and CSC (Finland); DLR and MPG (Germany); CSA (Canada); DTU Space
(Denmark); SER/SSO (Switzerland); RCN (Norway); SFI (Ireland); FCT/MCTES (Portugal); and PRACE (EU). A detailed description of the Planck Collaboration and a list of its members can be found at http://www.rssd.esa.int/index.php?project=PLANCK \&page $=$ Planck_Collaboration.

\section{REFERENCES}

Banday, A. J., et al., 2003, Reappraising Foreground Contamination in the COBE-DMR Data, MNRAS, 345, 897

Davies, R. D., et al., 1987, Sensitive Measurement of Fluctuations in the Cosmic Microwave Background, Nature, 326, 462

De Oliveira-Costa, A., et al., 1997, Galactic Microwave Emission at Degree Angular Scales, ApJ, 482, L17

De Oliveira-Costa, A. \& Tegmark, M., 1999, Editors of the Microwave Foregrounds ASP Conference Series Vol. 181

De Oliveira-Costa, A., et al., 1999, Cross-Correlation of the Tenerife Data with Galactic Templates Evidence for Spinning Dust ?, ApJ, 527, L9

Dickinson, C., et al., 2003, Towards a Free-Free Template for CMB Foregrounds, MNRAS, 341, 369

Drain, B. T. \& Lazarian, A., 1998, Diffuse Galactic Emission from Spinning Dust Grains, ApJ, 494, L19

Erickson, W. C., 1957, A Mechanism of Non-Thermal Radio-Noise Origin, ApJ, 126, 480

Finkbeiner, D. P., et al., 2002, Tentative Detection of Electric Dipole Emission from Rapidly Rotating Dust Grains, ApJ, 566, 898

Gaustad, J. E., et al., 2001, A Robotic Wide-Angle H-alpha Survey of the Southern Sky, PASP, 113, 1326

Haslam, C. G. T., et al., 1982, A 408 MHz All-Sky Continuum Survey. II - The Atlas of Contour Maps, A\&AS, 47, 1

Hoang, T., et al., 2010, Improving the Model of Emission from Spinning Dust: Effects of Grain Wobbling and Transient Spin-Up, ApJ, 715, 1462

Kogut, A., et al., 1996, Microwave Emission at High Galactic Latitudes in the Four-Year DMR Sky Maps, ApJ, 464, L5

Kogut, A., 1999, Anomalous Microwave Emission, in Microwave Foregrounds, ASP Conference Series Vo. 181, Eds. A. de Oliveira-Costa and M. Tegmark

Lagache, G., 2003, The Large-Scale Anomalous Microwave Emission Revisited by WMAP, A\&A, 405,813 
Leitch, E. M., et al., 1997, An Anomalous Component of the Galactic Emission, ApJ, 486, L23

Martin, P. G., 1972, On the Interaction of Rotating Interstellar Grains with Cosmic Lowfrequency Radiation, MNRAS, 155, 283

Planck Collaboration, 2011a, Planck Early Results XX. New Light on Anomalous Microwave Emission from Spinning Dust Grains, A\&A, 536, A20

Planck Collaboration, 2011b, Planck Early Results XXI. Properties of the Interstellar Medium in the Galactic Plane, A\&A, 536, A21

Reynolds, R. J., et al., 1998, The Wisconsin H-alpha Mapper (WHAM): A brief Review of Performance Characteristics and Early Scientific Results, PASA, 15, 14

Silsbee, K., et al., 2011, Spinning Dust Emission: The Effect of Rotation around a Non-Principal Axis, MNRAS, 411, 2750

Rouan, D., 1992, Physics of the Rotation of a PAH Molecule in Interstellar Environments, A\&A, 253, 498

Tauber, J, et al., 2001, Planck Pre-Launch Status: The Planck Mission, A\&A, 520, A1

Ysard, N. \& Verstraete, L., 2010, The LongWavelength Emission of Interstellar PAHs: Characterizing the Spinning Dust Contribution, A\&A, 509, A12 